\title{
The impact of positive peritoneal cytology on prognosis in patients with cervical cancer: a meta-analysis
}

\author{
Sang-Hee Yoon ${ }^{1}$, Soo-Nyung Kim ${ }^{*}{ }^{1}$, Seung-Hyuk Shim ${ }^{1}$, Ji-Young Lee ${ }^{1}$, Sun-Joo Lee ${ }^{1}$, In-Kyeong Oh ${ }^{1}$, \\ Hyeon-Jeong $\mathrm{Kim}^{1}$ and Soon-Beom Kang ${ }^{1}$ \\ ${ }^{1}$ Department of Obstetrics and Gynecology, Research Institute of Medical Science, Konkuk University School of Medicine, Seoul, \\ Korea
}

Background: The impact of positive peritoneal cytology on the prognosis of cervical cancer is controversial. Thus, we performed a meta-analysis to determine its impact on recurrence, and to investigate correlations between abnormal cytology and/or lymph node metastasis in cervical cancer.

Methods: A systematic literature review was conducted through July 2014. Odds ratios (ORs) and their $95 \%$ confidence intervals $(95 \% \mathrm{Cls})$ were calculated by standard meta-analysis techniques with the fixed-effects models, if there was no significant statistical heterogeneity across studies by using $l^{2}$.

Results: Of 303 studies retrieved, 6 were included in the meta-analysis. These six case-control observational studies included 1360 cervical cancer patients who showed negative peritoneal cytology and 64 who showed positive peritoneal cytology. Over the combined study period, 20 of 45 in the positive peritoneal cytology group experienced recurrence, whereas 88 of 539 controls did. The meta-analysis based on the fixed-effects model indicated a significant increase in the risk of recurrence in the positive peritoneal cytology group relative to the control group (OR: $4.47 ; 95 \% \mathrm{Cl}: 2.33-8.58, P<0.001, I^{2}=0.0 \%$ ). Moreover, the results of our meta-analysis suggested that the positive peritoneal cytology group displayed more lymph node metastasis than the negative peritoneal cytology group (OR: 3.73; 95\% Cl: $2.13-6.53, P<0.001, I^{2}=0.0 \%$ ).

Conclusions: Although based mainly on retrospective observational studies, our meta-analysis indicates that abnormal peritoneal cytology may be strongly associated with poor prognosis in patients with cervical cancer. Future research should verify this relationship through prospective observational studies over a longer term.

Peritoneal cytology examination is commonly performed for samples obtained during gynecology surgery. The prognostic value of peritoneal cytology in ovarian cancer among gynaecological neoplasms is widely accepted, and it is included in the International Federation of Gynecology and Obstetrics (FIGO) nomenclature (1994) (Benedet et al, 2003). In endometrial carcinoma, peritoneal cytology is included in the FIGO staging system, and positive cytology must be reported separately without changing the stage. A positive cytology result is considered a poorer prognostic factor. However, only a few reports have addressed the issue of positive peritoneal cytology in cervical neoplasms (Benedet et al, 2003; Kuji et al, 2014). In cervical cancer, the incidence of positive peritoneal cytology has been reported to be 0-15\% (Kilgore et al, 1984; Delgado et al, 1989; Takeshima et al, 1997; Estape et al, 1998; Kasamatsu et al, 2009). It is reported that the rate of positive peritoneal cytology among patients with squamous cell carcinoma (SCC) in FIGO stage I or II disease is low (0.3-1.8\%) (Delgado et al, 1989; Takeshima et al, 1997; Estape et al, 1998). On the other hand, positive peritoneal cytology was found four times more frequently in adenocarcinoma (ADC) than in SCC (Imachi et al, 1987). However, the prognostic value of peritoneal cytology in cervical carcinoma remains unanswered.

*Correspondence: Professor S-N Kim; E-mail: snkim@kuh.ac.kr

Received 17 February 2015; revised 12 June 2015; accepted 25 June 2015; published online 30 July 2015

(c) 2015 Cancer Research UK. All rights reserved 0007-0920/15 
Previous reports have been inconsistent; whereas one study failed to show any prognostic inference of positive peritoneal cytology in cervical cancer (Abu-Ghazaleh et al, 1984; Delgado et al, 1989; Trelford et al, 1995; Takeshima et al, 1997), other studies reported that patients with positive peritoneal cytology have a poor prognosis than those with negative cytology (Imachi et al, 1987; Ito and Noda, 1992; Kashimura et al, 1997; Estape et al, 1998; Zuna et al, 2009). Furthermore, some studies reported that positive peritoneal cytology was associated with poor prognosis only in the patients with ADC or adenosquamous carcinoma, but not in those with SCC (Kasamatsu et al, 2009; Kuji et al, 2014).

Therefore, we performed a meta-analysis using relevant studies to investigate the impact of positive peritoneal cytology on prognosis, focusing on lymph node metastasis and experienced recurrence, in cervical carcinoma when compared with negative peritoneal cytology.

\section{MATERIALS AND METHODS}

Search strategy. Two of the authors of the present study (S-HY and S-HS) designed the protocol and extraction forms in accordance with the Preferred Reporting Items for systematic Review and Meta-analyses (PRISMA) guideline. For this metaanalysis, we searched online abstracts from PubMed, Embase, and the Cochrane Central Register of Controlled Trials (CENTRAL) in the Cochrane Library through July 2014. For this search, we used the following keywords: 'cervical neoplasm or cancer or carcinoma or malignancy,' 'uterine cervical neoplasm or cancer or carcinoma or malignancy,' 'carcinoma of the cervix or the uterine cervix,' 'invasive carcinoma of the cervix or the uterine cervix,' 'squamous cell carcinoma of the cervix or the uterine cervix,' 'adenocarcinoma of the cervix or the uterine cervix,' and 'positive intra-peritoneal cytology or positive peritoneal washing cytology or abnormal cytology.' The titles and abstracts were checked to exclude any clearly unrelated articles. The full text of the remaining papers was evaluated to determine their relevance. In addition, the references cited in the selected papers and published reviews were checked to evaluate whether they included any additional studies of relevance.

Selection criteria. Studies were included if (1) they were randomised controlled trials, a prospective or retrospective cohort study, a population-based case-control study, or a nested casecontrol study; (2) the participants of interest received surgical treatment for cervical cancer; (3) the intervention of interest was peritoneal cytology; (4) the outcome measured was cervical cancer recurrence and/or lymph node metastasis, measured as the relative risk, the odds ratio (OR), or the hazard ratio estimated with the 95\% confidence interval (CI) (or sufficient data for calculating them). Single-arm cohort studies were not included in the metaanalysis. The studies that included advanced stage disease, such as stage III or IV, were excluded from the meta-analysis.

Data extraction. Data from each included study were systematically extracted by two authors using a standardised form. The form documented the most relevant items, including the name of the first author, the year of publication, the study design, the study period, age, sample size (cases and controls or cohort size), tumour stage, histological type, peritoneal cytology positivity, follow-up period, and prognostic value (recurrence, lymph node metastasis) between positive and negative peritoneal cytology.

Quality assessment. Study quality was evaluated independently by two authors using the nine-star Newcastle-Ottawa scale (NOS) criteria (Stang, 2010). The NOS criteria included three categories: (1) selection: $0-4$; (2) comparability: 0-2; (3) exposure (casecontrol studies) or outcomes (cohort studies): $0-3$. NOS scores ranged from 0 to 9 , with a score $\geqslant 7$ indicating good quality. Any disagreement was resolved after a discussion and reevaluation with the third author.

Statistical analysis. From the original study data, the OR and the 95\% CI were calculated for each study for the recurrence rate for positive and negative peritoneal cytology (Higgins and Thompson, 2002). Heterogeneity across studies was examined using $I^{2}$, which measures the percentage of the total variation across studies. Here substantial heterogeneity was defined as an $I^{2}$-value $>50 \%$ (Higgins et al, 2003). In the absence of significant heterogeneity, a fixed-effects model was used. When there was statistical heterogeneity, a random-effects model was used to estimate the combined OR for randomised and observational studies. Then, a subgroup analysis was conducted for the type of histology (SCC, ADC, or all type). The subgroup analysis was planned a priori before the data were collected and analysed. To evaluate the influence of single studies on the overall estimate, a sensitivity analysis was performed. Publication bias was evaluated using a graphical method. A funnel plot was built to assess this bias by using the s.e. and the OR (Sterne and Egger, 2001; Sterne et al, 2001). Publication bias was evaluated using the Begg-Mazumdar rank correlation test, Egger's test and the fail-safe $N$-test (Orwin and Boruch, 1983; Begg and Mazumdar, 1994). Comprehensive Meta-Analysis version 2.0 (Biostat, Englewood, NJ, USA) was used for all statistical tests. $P<0.05$ was considered significant for this meta-analysis. Data from this meta-analysis are presented according to the checklist based on the Meta-analysis Of Observational Studies in Epidemiology (Stroup et al, 2000).

\section{RESULTS}

Literature search. Figure 1 presents a flow diagram showing how relevant studies were identified. A total of 22 potentially relevant papers were found by focusing on abnormal peritoneal cytology and prognosis, particularly recurrence or lymph node metastasis (Creasman and Rutledge, 1971; Keettel et al, 1974; Hughes et al, 1980; Abu-Ghazaleh et al, 1984; Kilgore et al, 1984; Ziselman et al, 1984; Willett, 1985; Roberts et al, 1986; Imachi et al, 1987; Delgado et al, 1989; Zuna et al, 1990; Ito and Noda, 1992; Morris et al, 1992; Patsner, 1992; Trelford et al, 1995; Zuna and Behrens, 1996; Zuna, 1996; Kashimura et al, 1997; Takeshima et al, 1997; Estape et al, 1998; Kasamatsu et al, 2009; Kuji et al, 2014). Among these 22 papers, 16 were excluded: seven papers were incomplete studies providing no exact recurrence data (Abu-Ghazaleh et al, 1984; Kilgore et al, 1984; Zuna et al, 1990; Morris et al, 1992; Patsner, 1992; Trelford et al, 1995; Estape et al, 1998), one paper was a letter to the editor (Zuna, 1996), and another five studies lacked relevance to prognosis and cervical cancer (Creasman and Rutledge, 1971; Hughes et al, 1980; Ziselman et al, 1984; Willett, 1985; Zuna and Behrens, 1996); three studies included patients who underwent surgical treatment at advanced stages (Keettel et al, 1974; Roberts et al, 1986; Imachi et al, 1987). The remaining six studies reported on abnormal peritoneal cytology and prognosis and, thus, were included in the meta-analysis (Delgado et al, 1989; Ito and Noda, 1992; Kashimura et al, 1997; Takeshima et al, 1997; Kasamatsu et al, 2009; Kuji et al, 2014).

Study characteristics. After the final screening of the six relevant retrospective case-control observational studies, 1424 patients were enrolled in this meta-analysis, including a total of 64 cervical cancer patients with positive peritoneal cytology and 1360 with negative cytology. Publication years of the eligible studies ranged from 1989 to 2014. The studies were from the United States and Japan, and were assessed to range from 7 to 8 on the nine-star NOS. Table 1 shows the study characteristics in detail.

All of the patients had FIGO stage IA-IIB disease. Most histologic types, in particular, SCC, ADC and adenosquamous 


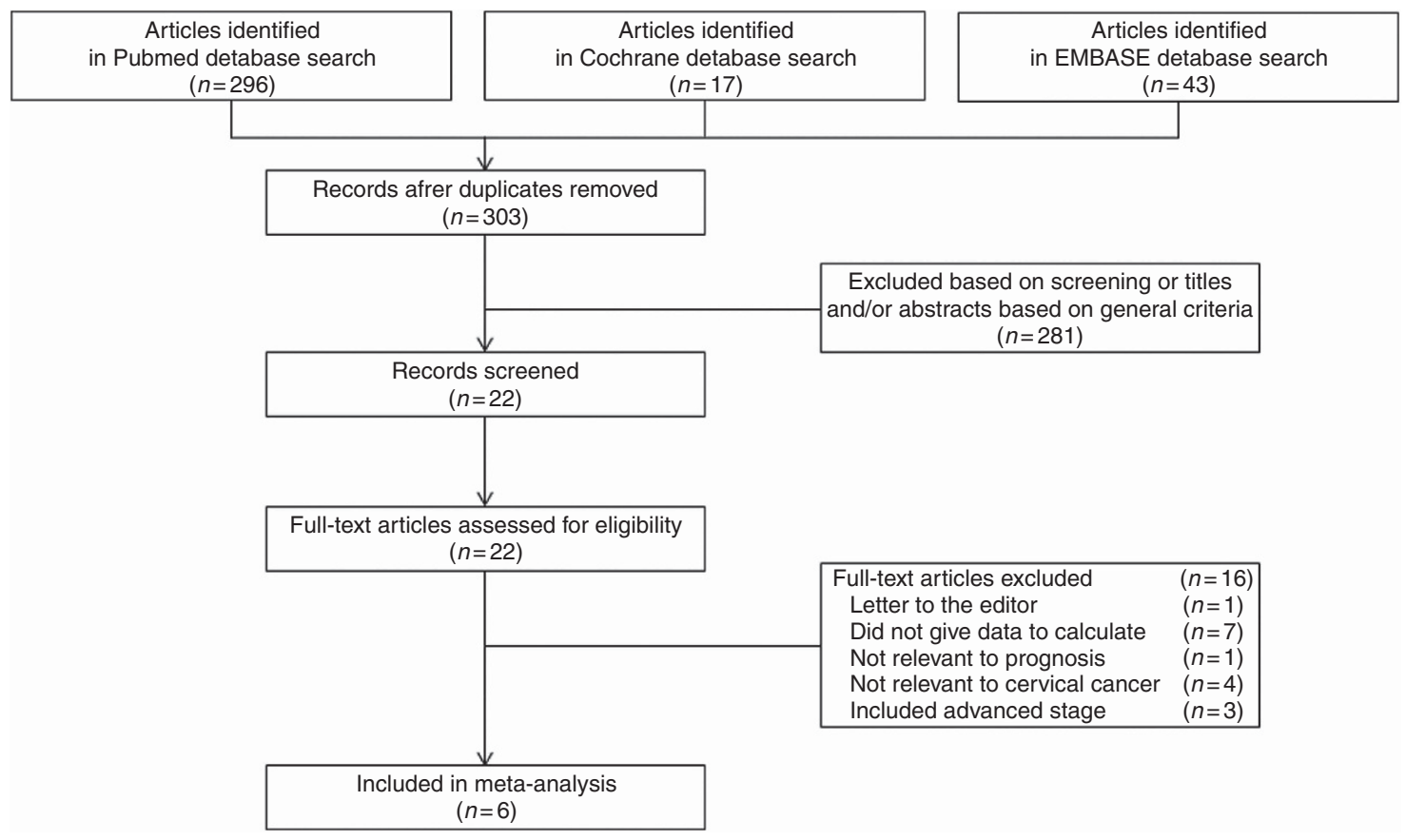

Figure 1. Flow diagram of the procedure for the literature search.

carcinoma, were included in each study, with the exception of two studies. Specifically, one study included only ADC histologic type (Kasamatsu et al, 2009) and another, only SCC (Delgado et al, 1989). Among all patients, 1360 (95.5\%) showed negative peritoneal cytology, whereas positive peritoneal cytology was observed in $64(4.5 \%)$.

Meta-analysis. Three studies (Ito and Noda, 1992; Takeshima et al, 1997; Kasamatsu et al, 2009) included a total of 584 patients with a combined total of 108 recurrences ( 20 of which occurred in the 45 patients with positive peritoneal cytology). Figure $2 \mathrm{~A}$ shows the ORs for cervical cancer recurrence for each study, and for all studies combined, comparing the positive peritoneal cytology with negative cytology. As no heterogeneity existed among studies $\left(P=0.473\right.$ and $\left.I^{2}=0 \%\right)$, the fixed-effects model was used. The pooled OR for positive peritoneal cytology and the risk of cervical cancer recurrence was 4.468 (95\% CI: 2.326-8.583, $P<0.001$ ).

There were six studies (Delgado et al, 1989; Ito and Noda, 1992; Kashimura et al, 1997; Takeshima et al, 1997; Kasamatsu et al, 2009; Kuji et al, 2014), including 1424 patients with a combined total of 317 lymph node metastases (34 of which occurred in the 64 patients with positive peritoneal cytology). Figure 2B shows the ORs for lymph node metastasis for each study, and for all studies combined, comparing positive peritoneal cytology with negative cytology. As no heterogeneity existed among studies $(P=0.606$ and $\left.I^{2}=0 \%\right)$, the fixed-effects model was used. The pooled OR for positive peritoneal cytology and the risk of lymph node metastasis was 3.726 (95\% CI: 2.127-6.525, $P<0.001$ ).

In the sensitivity analysis, the results based on the omission of one study at a time and the calculation of the pooled OR for the remaining studies showed that no study had a significant effect on the pooled OR (Figure 3A and B).

Subgroup analysis by the type of histology. Figure $4 \mathrm{~A}$ and $\mathrm{B}$ shows the OR for each study and the pooled OR for categories of ADC (including adenosquamous carcinoma), SCC, and ADC (including adenosquamous carcinoma) plus SCC. Positive peritoneal cytology in ADC-type cervical cancer led to a significant increase in recurrence (OR: 3.684; 95\% CI: $1.680-8.077 ; P=0.001$ and $\left.I^{2}=0 \%\right)$. Positive peritoneal cytology in cervical cancer with the ADC plus SCC histological type was also associated with recurrence (OR: 9.667; 95\% CI: 2.132-43.829; $P=0.003$ and $I^{2}=0 \%$ ). Although only one study (Takeshima et al, 1997) reported on the SCC histological type (OR: 1.701; 95\% CI: $0.172-16.780 ; P=0.649$ and $\left.I^{2}=0 \%\right)$, there was no association between positive peritoneal cytology and recurrence (Figure 4A).

Positive peritoneal cytology in ADC-type cervical cancer was associated with a significant increase in lymph node metastasis (OR: 2.435; 95\% CI: $1.088-5.450 ; P=0.030$ and $I^{2}=0 \%$ ). Positive peritoneal cytology in cervical cancer with ADC plus SCC histological type was also associated with lymph node metastasis (OR: 7.024; 95\% CI: 2.422-20.368; $P<0.001$ and $I^{2}=0 \%$ ). As for the SCC histological type, there were two studies (OR: 4.224; 95\% CI: $1.343-13.288 ; P=0.014$ and $I^{2}=0 \%$ ) (Figure 4B) (Delgado et al, 1989; Kashimura et al, 1997).

These results suggest that positive peritoneal cytology is associated with a higher risk of recurrence, especially for the $\mathrm{ADC}$ or adenosquamous carcinoma histological types, and with higher rates of lymph node metastasis, regardless of histological type.

Publication bias. A funnel plot for publication bias was slightly asymmetric (Figure 5A). This asymmetry could be caused by small study effects. The Begg and Mazumdar rank correlation test showed no significance $(P=0.296)$. The Begg's test presents some important limitations. A significant correlation suggests that a bias exists, but does not directly address the implications of this bias. Conversely, a non-significant correlation may be due to low statistical power, and cannot be considered as evidence that bias is absent. In this study, the Egger's test showed significance $(P=0.038)$. The classic fail-safe $N$-method can address the concerns that the entire observed effect may be an artefact of bias. This meta-analysis incorporates data from three studies, which yielded a $z$-value of 4.64 and a corresponding two-tailed $P$-value of 0.00001 . The fail-safe $N$ is 14 . This means that we would need to locate and include 14 'null' studies in order for the combined two-tailed $P$-value to exceed 0.050 . However, a funnel plot for publication bias regarding LN metastasis was almost symmetric (Figure 5B). 
Table 1. Characteristics of studies included in the meta-analysis

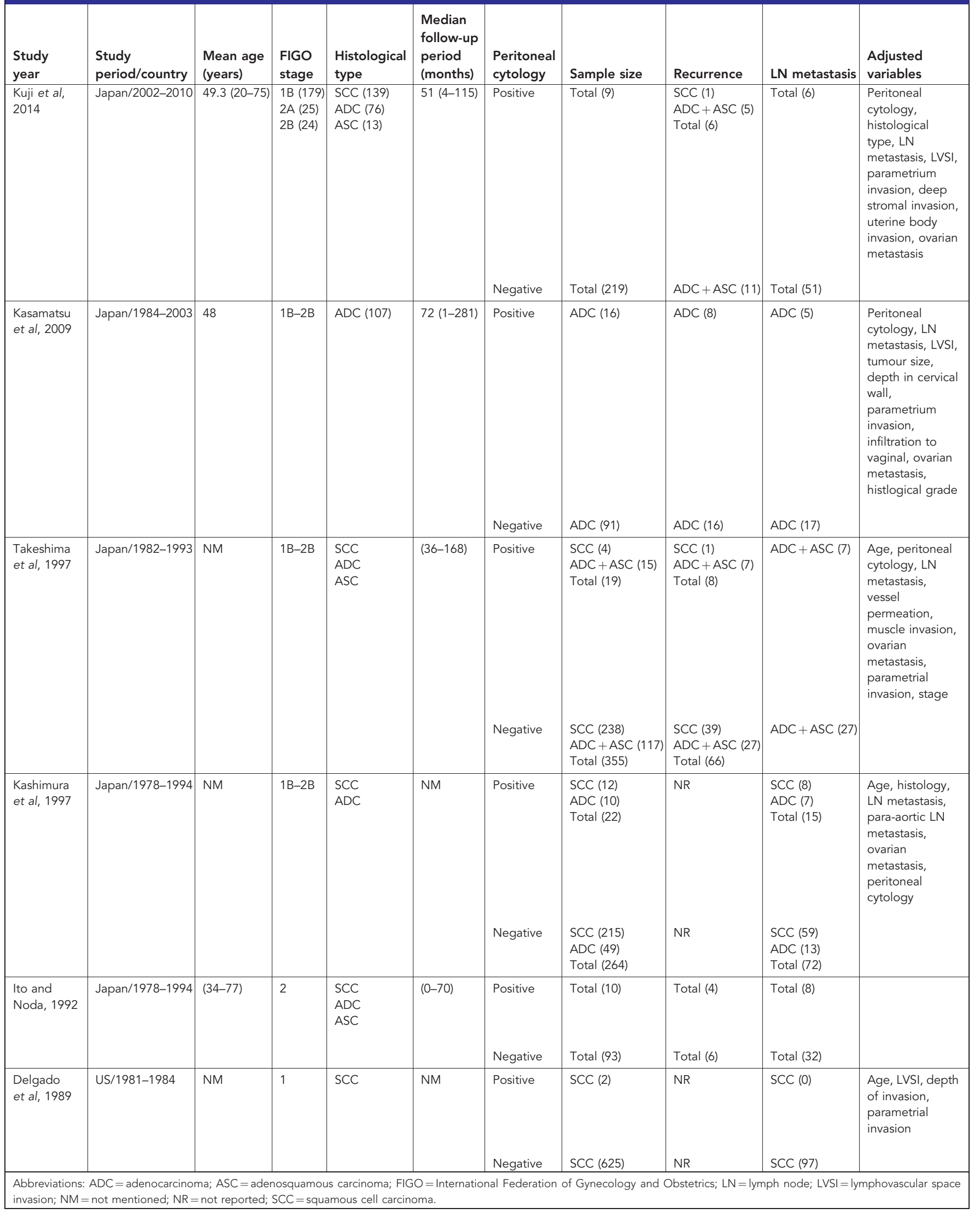




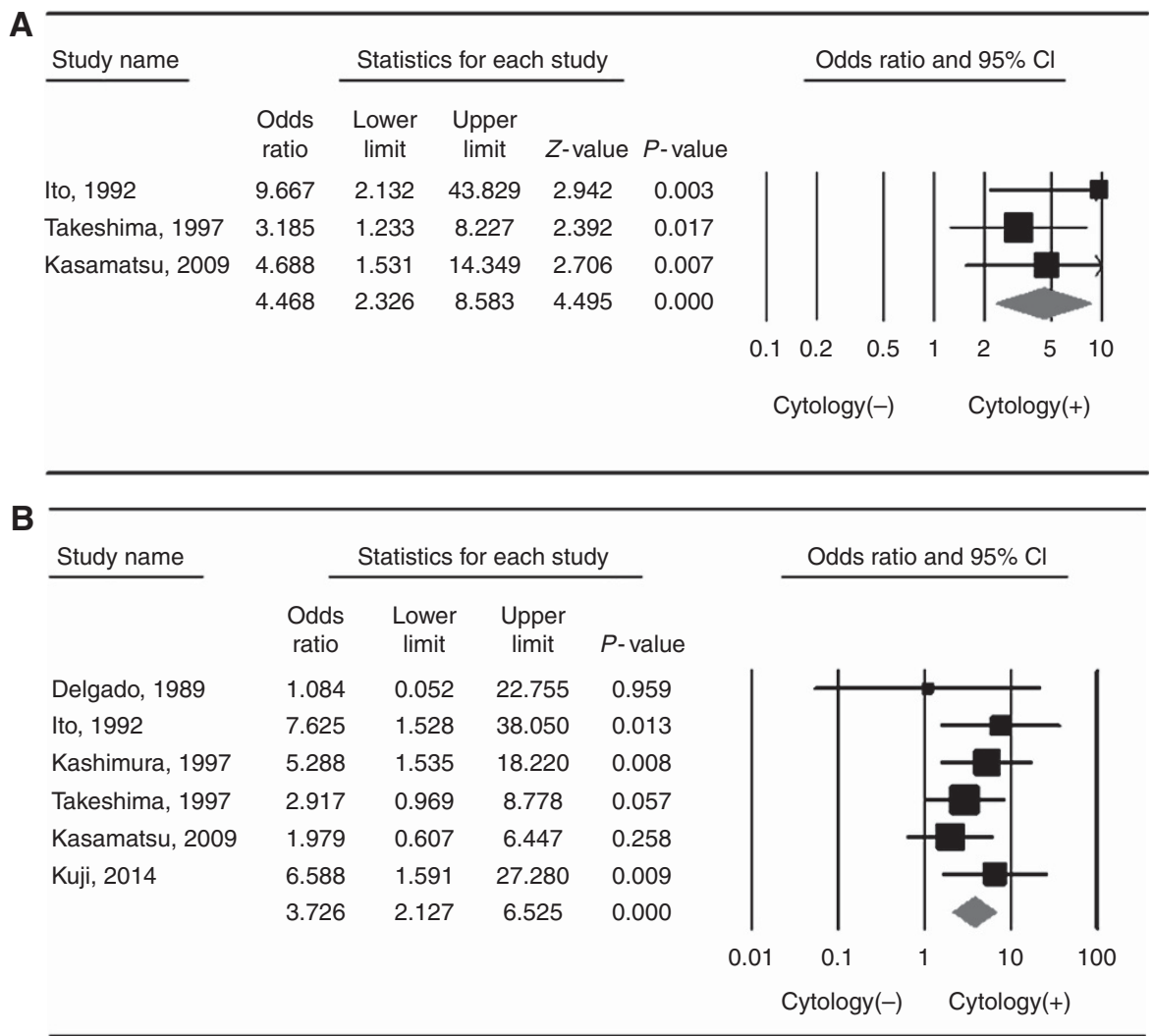

Figure 2. The odds ratio for the risk of (A) recurrence and (B) LN metastasis for each study and all studies combined comparing positive peritoneal cytology with negative cytology in a meta-analysis based on the fixed-effects model. Heterogeneity was low across studies (A: $P=0.473$ and $I^{2}=0 \% ; B: P=0.606$ and $P^{2}=0 \%$ ). The size of each square is proportional to the sample size for each study, and the horizontal line through the square indicates the $95 \%$ confidence interval for that study. For the pooled analysis, the diamond indicates the pooled value, and the right and left ends of the diamond indicate the $95 \%$ confidence interval for the analysis.

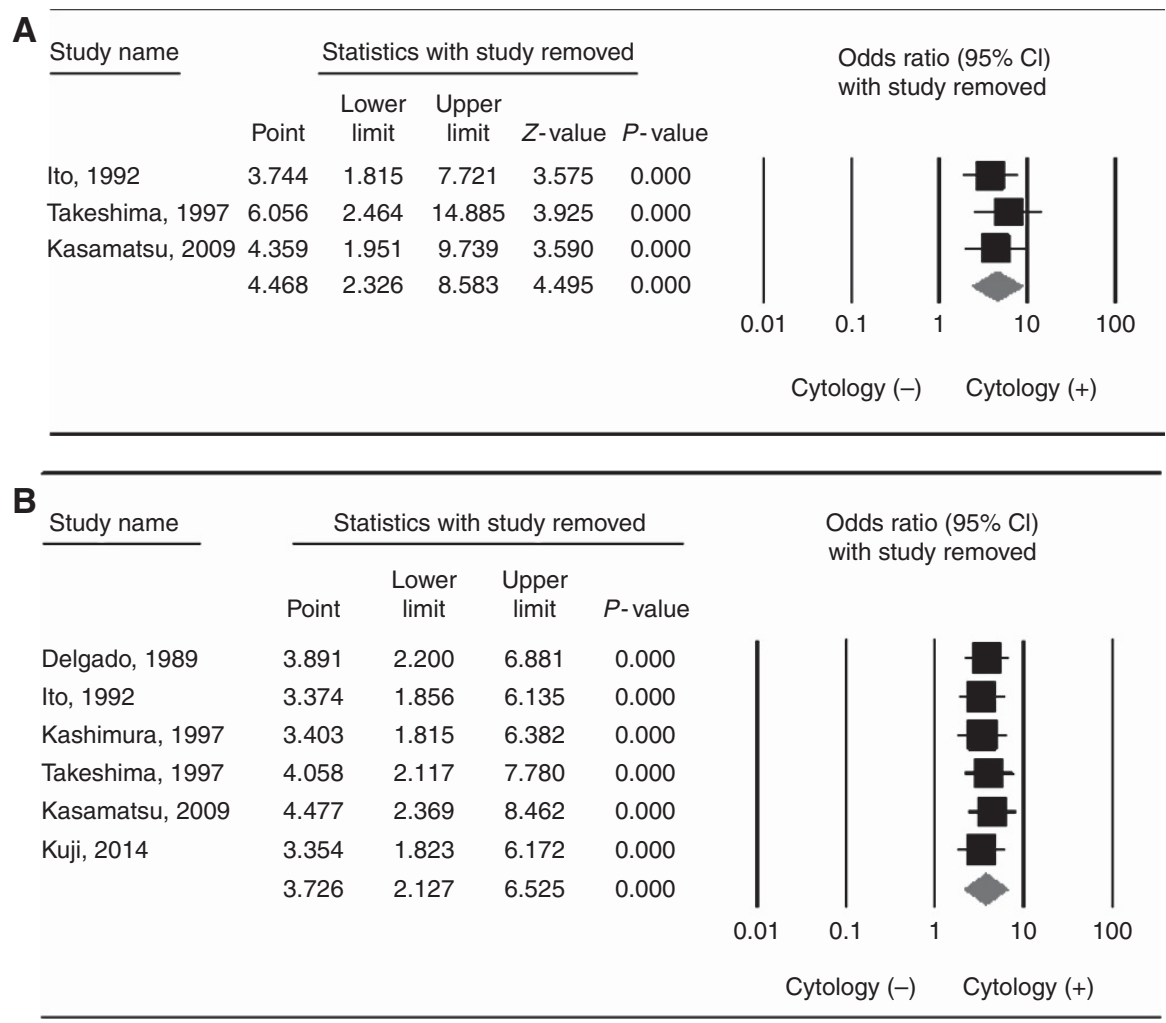

Figure 3. Sensitivity analysis of the summary odds ratio coefficients on the relationships between abnormal peritoneal cytology and prognosis (A: recurrences, B: LN metastasis) of patients with cervical cancer. 
A

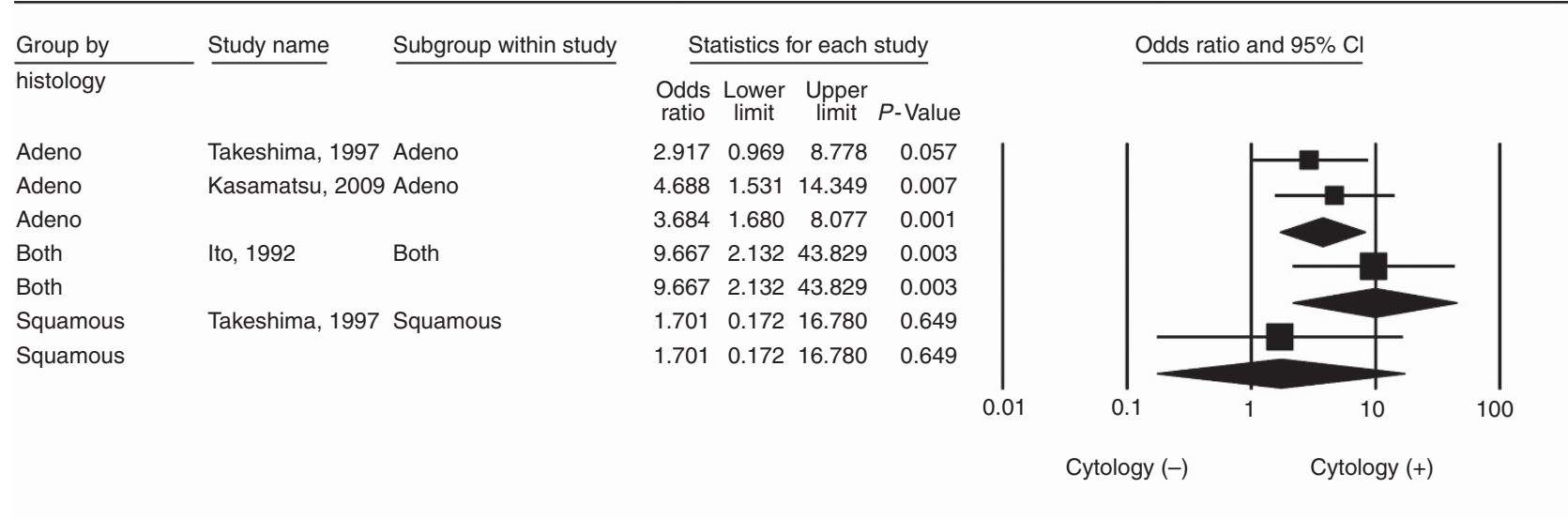

B

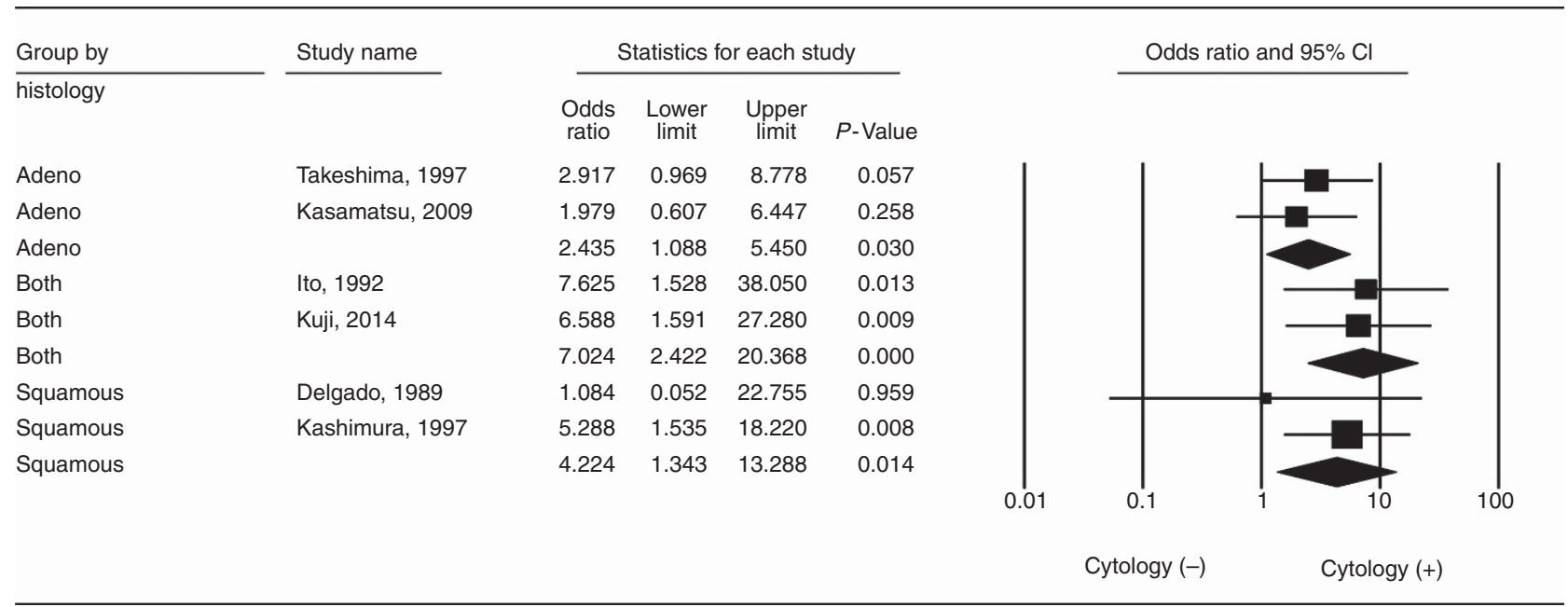

Figure 4. Positive peritoneal cytology and the risk of (A) recurrence and (B) LN metastasis in the subgroup meta-analysis by histological type, based on the fixed-effects model.

\section{DISCUSSION}

According to published data, patients with positive peritoneal cytology had poorer prognostic outcomes, such as survival rates and recurrence, than those with negative cytology. However, the sample size in most studies was not large enough to sufficiently explain the effectiveness of cytology on cervical cancer prognosis, and no quantitative analysis has estimated the efficacy of cytology on cervical cancer prognosis. Moreover, it is well known that, positive peritoneal cytology overlaps with other risk factors. Roberts et al (1986) reported that positive peritoneal cytology was associated with a very poor prognosis, and it was also associated with other poor prognostic factors (Roberts et al, 1986). Thus, no consensus was reached as to whether positive peritoneal cytology is an independent risk factor. Takeshima et al (1997) found that peritoneal cytology was not a prognostic factor for stage I and II ADC, although cardinal ligament invasion, lymph node metastases, and muscle layer invasion were prognostic factors (Takeshima et al, 1997). Kasamatsu et al (2009) found that peritoneal cytology, para-aortic lymph nodes, and pelvic lymph nodes were independent prognostic factors, irrespective of the histological type (Kasamatsu et al, 2009). Kuji et al (2014) reported that positive peritoneal cytology may be associated with a poor prognosis in adenosquamous carcinoma or ADC of cervix (Kuji et al, 2014). The results of the present meta-analysis indicate that positive peritoneal cytology of cervical cancer patients leads to a significant increase in the risk of cervical cancer recurrence. However, the results of the subgroup analysis based on the histology show slightly different patterns. The estimates from the $\mathrm{ADC}$ histology type indicate that positive peritoneal cytology leads to an increase in the risk of recurrence (OR: 3.684; 95\% CI: $1.680-$ 8.077; $P=0.001$ ), whereas those from the squamous cell type did not lead to a significant increase in the risk of recurrence (OR: $1.701 ; 95 \%$ CI: $0.172-16.780 ; P=0.649)$. This finding might suggest the possibility that positive peritoneal cytology increases disease recurrence by the spread of viable tumour cells. If we can conclude that positive peritoneal cytology, particularly for patients with ADC, leads to recurrence by peritoneal dissemination through hematogenous or lymphatic spread, then positive peritoneal cytology would indicate systemic disease. According to the qualitative analysis, positive results of peritoneal cytology seem to be associated with high recurrence rates through peritoneal dissemination. The authors reported that the incidence of peritoneal spread at the first recurrence in the positive cytology group $(62.5 \%)$ was significantly higher than that in the negative cytology group (12.5\%) (Kasamatsu et al, 2009). In addition, another study reported that peritoneal recurrence of ADC among patients with positive peritoneal cytology occurred in $60 \%$ of cases (Kuji et al, 2014). This percentage tended to be higher than that in patients with negative cytology. However, in the present metaanalysis, we could not conduct a quantitative meta-analysis on the 

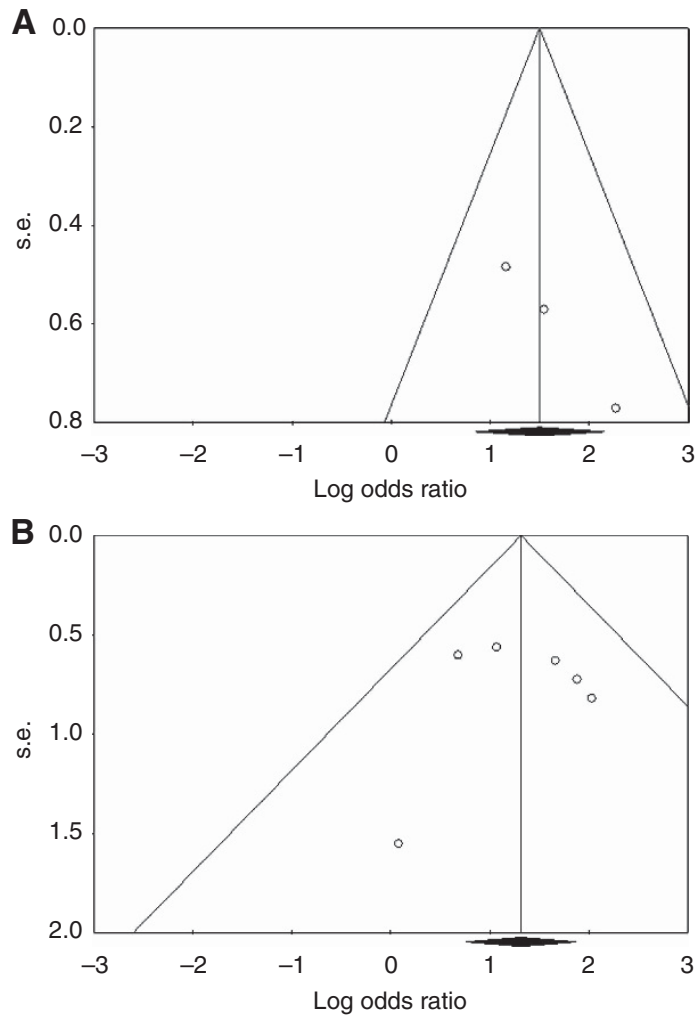

Figure 5. Assessment of publication bias using funnel plot analysis. (A) Funnel plot analysis of studies on recurrence. The funnel plot has a asymmetrical distribution, suggesting the presence of selection bias.

(B) Funnel plot analysis of studies on LN metastasis. The Funnel plot has a almost symmetrical distribution. Publication bias was not found in the meta-analysis of $L N$ metastasis.

relationship between abnormal peritoneal cytology and metastatic pattern including peritoneal dissemination, because we could not obtain detailed individual data. Considering the above facts, further investigation and well-designed randomised controlled trials may be needed to reach a definitive conclusion.

Delgado et al (1989) found that the factors identified as independent risk factors for pelvic lymph node metastasis by multivariate analysis were depth of invasion, parametrium involvement, lymph-vascular space involvement, and age, but not peritoneal cytology (Delgado et al, 1989). Moreover, Ito and Noda (1992) reported that negative cytology was often found even in cases with metastasis to retroperitoneal lymph nodes, whereas positive cytology was found in cases without metastasis (Ito and Noda, 1992). These authors suggested that the mechanism of cervical carcinoma metastasis to retroperitoneal lymph nodes might not be the same as that of spreading into the abdominal cavity. However, Kashimura et al (1997) reported that peritoneal cytology, lymph node metastasis, ovarian metastasis, and histological grade are independent prognostic factors in stage I and stage II ADC of the cervix (Kashimura et al, 1997). The results of our meta-analysis indicate that positive peritoneal cytology of cervical cancer patients leads to a significant increase in the risk of lymph node metastasis. This pattern was also observed in the subgroup analysis for histological type. The estimates from the ADC histology type and SCC type indicate that positive peritoneal cytology leads to an increase in the risk of lymph node metastasis (OR: 2.435; 95\% CI: $1.088-5.450 ; P=0.030$, and OR: $4.224 ; 95 \%$ CI: $1.343-13.288 ; P=0.014$, respectively). This finding could present, whatever histology type of cancer was, significant increase in lymph node metastasis was found between the peritoneal cytology positive and cytology negative groups.
Our meta-analysis results revealed that positive peritoneal cytology was associated with recurrence in histology type including ADC or adenosquamous carcinoma and lymph node metastasis regardless of histological type. Therefore, it is crucial to obtain peritoneal cytology fluid routinely at the time of operation in patients with early-stage cervical cancer, especially ADC histological type. Although we performed adjuvant treatment such as postoperative concurrent chemoradiotherapy or radiotherapy according to the risk factors, if positive peritoneal cytology truly reflects peritoneal spread or affects survival adversely, we think that systemic chemotherapy could be considered as the treatment of choice for patients with positive peritoneal cytology, especially for those with the ADC histological type, for similar reasons regarding peritoneal cytology as a risk factor of endometrial and ovarian cancers (Rahaman et al, 2009; Berek, 2012a,b). Patients with positive cytology along with poor prognostic factors may potentially benefit from systemic chemotherapy in endometrial cancer (Berek, 2012b).

Nevertheless, several limitations of the present meta-analysis should be recognised. First, as our meta-analysis was a retrospective study, it may have led to subject selection bias, which could have influenced the reliability of our results. Second, this meta-analysis was based on data published from the included studies, and we could not obtain detailed individual data. Thus, we were not able to correct all clinicopathological parameters into a consistent standard, for example, stage, risk factors (lymph node status, parametrial invasion, operative margin status, tumour size, lymphovascular space invasion, and deep stromal invasion), treatment method (e.g., radical operation field scale or adjuvant treatment method), follow-up period, recurrence diagnostic method, and recurrent pattern. Therefore, it was not possible to perform multivariate analysis and to determine the independent associated risk factor of poor prognosis among patients with positive peritoneal cytology and lymph node involvement in the present meta-analysis. In addition, we could not control potentially relevant bias factors (i.e., age, and ethnic group). Finally, funnel plot analyses concerning publication bias were also performed to confirm the reliability of our research results. In our meta-analysis, the publication bias in positive peritoneal cytology with recurrence may exist, but not with lymph node metastasis in cervical cancer. Consequently, more studies should be performed so that they can be included in a meta-analysis.

Although our study has some limitations, we believed it is the first meta-analysis focusing on the relationships between peritoneal cytology and the prognosis of cervical cancer. Furthermore, we made an effort to perform a highly sensitive literature search strategy utilising electronic databases.

In summary, to our knowledge, our meta-analysis is the first report to show that positive peritoneal cytology may be strongly associated with poor prognosis in cervical cancer. Our meta-analysis indicates that peritoneal cytology may serve as a prognostic factor for cervical cancer. Thus, it is essential to obtain peritoneal cytology routinely at the time of laparotomy in patients with cervical cancer about histology type including $\mathrm{ADC}$ or adenosquamous carcinoma, especially. Moreover, the results of peritoneal cytology must be considered in postoperative treatment planning. Therefore, we propose that positive cytology should be reported separately without changing the stage, and suggest that positive peritoneal cytology is a risk factor that should be taken into account when making decisions concerning postoperative adjuvant therapy in patients with earlystage cervical cancer. When the result of peritoneal cytology is positive, additional aggressive postoperative chemotherapy may be proposed as an adjuvant therapy.

However, owing to the limitations acknowledged above, further research, with larger sample sizes and more amount of comprehensive data, is still required to provide a more representative statistical analysis. Therefore, well-designed randomised controlled trials or prospective cohort studies are needed in the future. 


\section{ACKNOWLEDGEMENTS}

This work was supported by the Konkuk University. This study received no financial support.

\section{CONFLICT OF INTEREST}

The authors declare no conflict of interest.

\section{REFERENCES}

Abu-Ghazaleh S, Johnston W, Creasman WT (1984) The significance of peritoneal cytology in patients with carcinoma of the cervix. Gynecol Oncol 17(2): 139-148.

Begg CB, Mazumdar M (1994) Operating characteristics of a rank correlation test for publication bias. Biometrics 50(4): 1088-1101.

Benedet JL, Odicino F, Maisonneuve P, Beller U, Creasman WT, Heintz AP, Ngan HY, Pecorelli S (2003) Carcinoma of the cervix uteri. Int J Gynaecol Obstet 83(Suppl 1): 41-78.

Berek JS (2012a) Berek \& Novak's Gynecology, 15th edn, vol. ch. 37. Lippincott Williams \& Wilkins: Philadelphia, PA, USA.

Berek JS (2012b) Berek \& Novak's Gynecology, 15th edn. Lippincott Williams \& Wilkins: Philadelphia, PA, USA.

Creasman WT, Rutledge F (1971) The prognostic value of peritoneal cytology in gynecologic malignant disease. Am J Obstet Gynecol 110(6): 773-781.

Delgado G, Bundy BN, Fowler Jr WC, Stehman FB, Sevin B, Creasman WT, Major F, DiSaia P, Zaino R (1989) A prospective surgical pathological study of stage I squamous carcinoma of the cervix: a Gynecologic Oncology Group Study. Gynecol Oncol 35(3): 314-320.

Estape R, Angioli R, Wagman F, Madrigal M, Janicek M, Ganjei-Azar P, Penalver M, Averette H (1998) Significance of intraperitoneal cytology in patients undergoing radical hysterectomy. Gynecol Oncol 68(2): 169-171

Higgins JP, Thompson SG (2002) Quantifying heterogeneity in a metaanalysis. Stat Med 21(11): 1539-1558.

Higgins JP, Thompson SG, Deeks JJ, Altman DG (2003) Measuring inconsistency in meta-analyses. BMJ 327(7414): 557-560.

Hughes RR, Brewington KC, Hanjani P, Photopulos G, Dick D, Votava C, Moran M, Coleman S (1980) Extended field irradiation for cervical cancer based on surgical staging. Gynecol Oncol 9(2): 153-161.

Imachi M, Tsukamoto N, Matsuyama T, Nakano H (1987) Peritoneal cytology in patients with carcinoma of the uterine cervix. Gynecol Oncol 26(2): 202-207.

Ito K, Noda K (1992) Peritoneal cytology in patients with uterine cervical carcinoma. Gynecol Oncol 47(1): 76-79.

Kasamatsu T, Onda T, Sasajima Y, Kato T, Ikeda S, Ishikawa M, Tsuda H (2009) Prognostic significance of positive peritoneal cytology in adenocarcinoma of the uterine cervix. Gynecol Oncol 115(3): 488-492.

Kashimura M, Sugihara K, Toki N, Matsuura Y, Kawagoe T, Kamura T, Kaku T, Tsuruchi N, Nakashima H, Sakai H (1997) The significance of peritoneal cytology in uterine cervix and endometrial cancer. Gynecol Oncol 67(3): 285-290.

Keettel WC, Pixley EE, Buchsbaum HJ (1974) Experience with peritoneal cytology in the management of gynecologic malignancies. Am J Obstet Gynecol 120(2): 174-182.
Kilgore LC, Orr Jr JW, Hatch KD, Shingleton HM, Roberson J (1984)

Peritoneal cytology in patients with squamous cell carcinoma of the cervix. Gynecol Oncol 19(1): 24-29.

Kuji S, Hirashima Y, Komeda S, Tanaka A, Abe M, Takahashi N, Takekuma M (2014) The relationship between positive peritoneal cytology and the prognosis of patients with FIGO stage I/II uterine cervical cancer. J Gynecol Oncol 25(2): 90-96.

Morris PC, Haugen J, Anderson B, Buller R (1992) The significance of peritoneal cytology in stage IB cervical cancer. Obstet Gynecol 80(2): 196-198.

Orwin RG, Boruch RF (1983) RRT meets RDD: statistical strategies for assuring response privacy in telephone surveys. Public Opin Q 46(4): 560-571.

Patsner B (1992) Peritoneal cytology in patients with stage IB cervical cancer undergoing radical hysterectomy: limited value. Eur J Gynaecol Oncol 13(4): 306-308

Rahaman J, Steiner N, Hayes MP, Chuang L, Fishman D, Gretz Iii H (2009) Chemotherapy for Gynecologic cancers. Mt Sinal J Med 76: 577-588.

Roberts WS, Bryson SC, Cavanagh D, Roberts VC, Lyman GH (1986) Peritoneal cytology and invasive carcinoma of the cervix. Gynecol Oncol 24(3): 331-336.

Stang A (2010) Critical evaluation of the Newcastle-Ottawa scale for the assessment of the quality of nonrandomized studies in meta-analyses. Eur J Epidemiol 25(9): 603-605.

Sterne JA, Egger M (2001) Funnel plots for detecting bias in meta-analysis: guidelines on choice of axis. J Clin Epidemiol 54(10): 1046-1055.

Sterne JA, Egger M, Smith GD (2001) Systematic reviews in health care: Investigating and dealing with publication and other biases in metaanalysis. BMJ 323(7304): 101-105.

Stroup DF, Berlin JA, Morton SC, Olkin I, Williamson GD, Rennie D, Moher D, Becker BJ, Sipe TA, Thacker SB (2000) Meta-analysis of observational studies in epidemiology: a proposal for reporting. Meta-analysis Of Observational Studies in Epidemiology (MOOSE) group. JAMA 283(15): 2008-2012.

Takeshima N, Katase K, Hirai Y, Yamawaki T, Yamauchi K, Hasumi K (1997) Prognostic value of peritoneal cytology in patients with carcinoma of the uterine cervix. Gynecol Oncol 64(1): 136-140.

Trelford JD, Kinney W, Vogt P (1995) Positive peritoneal cytology in stage I carcinoma of the cervix. Acta Cytol 39(2): 177-179.

Willett GD (1985) Prognostic value of cytologic peritoneal washings. Clin Lab Med 5(2): 265-274.

Ziselman EM, Harkavy SE, Hogan M, West W, Atkinson B (1984) Peritoneal washing cytology. Uses and diagnostic criteria in gynecologic neoplasms. Acta Cytol 28(2): 105-110.

Zuna RE (1996) Peritoneal washing cytology in gynecologic cancer: long-term follow-up of 355 patients [Letter to the editor]. J Natl Cancer Inst $\mathbf{8 8}(23)$ : 1779 .

Zuna RE, Behrens A (1996) Peritoneal washing cytology in gynecologic cancers: long-term follow-up of 355 patients. J Natl Cancer Inst 88(14): 980-987.

Zuna RE, Hansen K, Mann W (1990) Peritoneal washing cytology in cervical carcinoma. Analysis of 109 patients. Acta Cytol 34(5): 645-651.

Zuna RE, Moore WE, Shanesmith RP, Dunn ST, Wang SS, Schiffman M, Blakey GL, Teel T (2009) Association of HPV16 E6 variants with diagnostic severity in cervical cytology samples of 354 women in a US population. Int J Cancer 125(11): 2609-2613.

This work is published under the standard license to publish agreement. After 12 months the work will become freely available and the license terms will switch to a Creative Commons AttributionNonCommercial-Share Alike 4.0 Unported License. 